\title{
Institutions et territoires du vin en France : le cas de l'A.O.C. Cahors
}

\author{
Institutions and territories in the French wine \\ industry: the Cahors case
}

\author{
Jean-Guillaume Ditter* et Joëlle Brouard
}

Groupe ESC Dijon Bourgogne, 29 rue Sambin - BP 50608 - 21006 Dijon

\section{Résumé}

Cet article se propose d'apporter un éclairage particulier à l'analyse économique des vignobles français en AOC (appellation d'origine contrôlée). À partir d'une perspective institutionnelle, on montre qu'un vignoble en AOC est une articulation entre des «terroirs géophysiques » et un «terroir institutionnel », ensemble d'institutions ayant cours sur le territoire que constitue le vignoble, l'AOC étant l'une de ces institutions. Les performances économiques du vignoble dépendront en conséquence du degré de proximité institutionnelle existant entre les différents agents favorisant une bonne articulation entre ces deux dimensions du terroir. Le cas de l'AOC Cahors nous offre un champ d'application concret de notre grille d'analyse.

(C) 2012 Lavoisier, Paris. Tous droits réservés.

\section{Summary}

This article aims to renew the economic analysis of French DPO vineyards. From an institutional perspective, we show that a DPO vineyard connects various "geophysical terroirs" and an "institutional terroir", i.e. a set of institutions covering the territory that constitutes the vineyards, including the DPO labels. The economic performance of the vineyard will therefore depend on the degree

\footnotetext{
*Adresse email : Jean-Guillaume.Ditter@escdijon.eu doi:10.3166/ges.14.303-325 @ 2012 Lavoisier, Paris. Tous droits réservés.
} 
of institutional proximity between the agents entailing a high quality relationship between these two dimensions of terroirs. The case of Cahors DPO will then make it possible to carry out a field specific study based on our analytical framework.

(C) 2012 Lavoisier, Paris. Tous droits réservés.

Mots clés : terroir, territoire, institutions, proximité institutionnelle, AOC, Cahors.

Keywords: terroir, territory, institutions, institutional proximity, DPO, Cahors.

\section{Introduction ${ }^{1}$}

La référence à l'origine du produit a longtemps été la solution la plus performante mise en œuvre par les acteurs (vignerons, négociants) de la filière vitivinicole française pour lutter contre les fraudes et imitations et, plus généralement, pour valoriser leurs produits et se différencier de la concurrence (Martin, 2004). Elle a trouvé son apogée dans les années 1930 avec la mise en place progressive du système des appellations d'origine contrôlée $(\mathrm{AOC})^{2}$, substituant une relation au lieu de production du vin dans sa dimension collective à la relation au vigneron ou négociant pris individuellement.

Ce modèle a fait le succès des vins français jusqu'au début des années 2000 où l'émergence des vins dits du « Nouveau Monde », identifiés par un cépage ou une marque plutôt que par leur origine, a remis sa suprématie en cause (Garcia-Parpet, 2001). Il a alors été critiqué pour sa complexité, son manque de lisibilité et la confusion entre "typicité » et «qualité » du produit entretenue auprès du consommateur, là où les producteurs du Nouveau Monde recherchent la simplicité et le lien au marché (Ditter, 2005; Corade, 2008). Mais le constat qui s'impose actuellement est plus nuancé car toutes les AOC ne sont pas touchées - ainsi Châteauneuf-du-Pape, les AOC du Jura, Sancerre - tandis que certains vins du nouveau monde sont à leur tour entrés en crise, ainsi en Australie.

Nous posons donc comme hypothèse que ce n'est pas tant l'existence du modèle qui est remise en question que ses modalités de mise en œuvre. Nous nous proposons ici d'envisager le problème en nous demandant dans quelle mesure la performance d'un vignoble classé en AOC tient à ses caractéristiques institutionnelles, en tant que combinaison entre un ou plusieurs terroirs, au sens géophysique du terme, et un «terroir-territoire », tel qu'il apparaît dans une perspective institutionnelle. Au cœur du modèle se trouve l'AOC elle-même, institution formelle qui articule le terroir géophysique et le terroir-territoire.

Cet article s'inscrit donc dans le champ de l'analyse institutionnelle et de ses développements spatiaux. Le territoire y est analysé comme un objet construit, un ensemble d'institutions au sein desquelles s'inscrivent les rapports entre acteurs économiques et qui influent sur la performance relative de l'appareil de production local. Pour étayer notre proposition, nous définirons tout d'abord l'objet de l'approche institutionnelle et son apport dans l'analyse des stratégies de la filière vitivinicole. Puis, nous montrerons com-

\footnotetext{
${ }^{1}$ Les auteurs souhaitent remercier Abdelillah Hamdouch pour ses commentaires et suggestions.

${ }^{2}$ On utilise dans cet article les termes traditionnels français «AOC » et « vin de pays » plutôt que leurs équivalents européens « appellation d'origine protégée » (AOP) et « indication géographique protégée » (IGP).
} 
ment cette approche institutionnelle permet d'apporter un éclairage nouveau aux notions de terroir, vignoble et territoire viticoles. Nous analyserons pour finir le cas particulier du vignoble cadurcien et de l'AOC Cahors.

\section{Institutions et territoires du vin}

\subsection{Institutions et territoire}

\subsubsection{L'approche institutionnelle}

On regroupe sous le terme d'approche institutionnelle un ensemble diversifié d'écoles de pensée au croisement de l'économie, de la sociologie ou de l'histoire, dont le point commun est de s'intéresser aux institutions et à leur impact sur les comportements et la coordination des acteurs économiques. Veblen (1899), qui les définit comme des " habitudes de pensée et d'action dominante au sein de la communauté sociale », en est l'initiateur généralement reconnu. Autre auteur majeur, Commons (1950) distingue les «transactions », interactions par lesquelles deux ou plusieurs individus s'échangent des droits de propriété, des «institutions », actions collectives qui contrôlent les actions individuelles. Un de ses apports est de différencier institutions informelles (la coutume) et institutions formelles, ces dernières étant pour lui des organisations, telles que l'État, les entreprises ou les syndicats.

Le courant dit de la «nouvelle économie institutionnelle », impulsé par Coase (1937) et structuré par Williamson (1985), va chercher à appliquer les outils de l'analyse institutionnelle à l'analyse des organisations. Williamson développe en particulier la théorie des coûts de transaction, "coûts de recherche, de négociation, de suivi et de répétition d'un contrat avec un partenaire économique », pour démontrer l'existence d'arrangements institutionnels, modes d'organisation des transactions, alternatifs au marché dans un environnement institutionnel donné. Il introduit la notion d'actif - ou ressource - spécifique, générateur de coûts de transaction. La notion même d'environnement institutionnel n'apparaît cependant que tardivement dans les travaux de Williamson et sa compréhension n'en est pas au cœur.

Notre approche des institutions se fonde sur celle de North (1990, 2005) qui, quoique rattaché à la nouvelle économie institutionnelle dont il mobilise les principaux concepts, s'en distingue en s'éloignant progressivement des standards de l'analyse néoclassique pour s'intéresser au changement institutionnel dans une perspective dynamique et historique. Il définit les institutions comme «les règles formelles ou des contraintes informelles et leurs modes de mise en auvre (enforcement) qui encadrent et régulent les comportements des acteurs économiques » et ont pour objet de simplifier les interactions entre les acteurs en limitant les possibilités d'interprétation et de confusion. Il différencie clairement les institutions, les règles du jeu, des organisations, qui en sont les « joueurs ».

L'impact des institutions peut être envisagé sous des angles différents. North identifie des institutions informelles implicites ou tacites, qui sont des normes sociales, conventions, habitudes personnelles, ou encore routines organisationnelles. Dans les sociétés complexes, elles sont complétées par des institutions formelles, qui sont explicites et se présentent sous forme de constitutions, lois, règlements, codes, dont la fonction principale est de coordonner et standardiser les interactions entre les acteurs au sein d'une 
société. Les modes de mise en œuvre sont essentiellement la crainte de l'exclusion, en ce qui concerne les institutions informelles, et la contrainte publique pour ce qui est des institutions formelles (Didry et Vincensini, 2010).

Scott (1995) distingue pour sa part les institutions en fonction de leur mode opérationnel. Les institutions coercitives sont définies comme un ensemble de règles et un système de sanctions qui contraignent le comportement des acteurs. Les institutions normatives guident celui-ci en définissant ce qui est attendu et/ou approprié selon les circonstances. Enfin, les institutions culturelles se composent d'un ensemble d'habitudes de pensée et de raisonnements auxquels sont associés des types de comportements.

Ménard (cité par Hamdouch (2005); p. 232) condense les principales acceptions de la notion d'institution en une définition synthétique : "An institution is manifested in a long-standing set of stable, abstract and impersonal rules, crystallized in traditions, customs, or laws, so as to implement and enforce patterns of behavior governing the relationships between separate social constituencies ». Nous adopterons cette définition, qui permet en particulier d'expliciter la distinction entre les institutions proprement dites (règles du jeu) et les arrangements institutionnels que sont les organisations, marchés et les structures de gouvernance.

Un questionnement central de l'approche institutionnelle est celui de la dynamique des institutions, à savoir les conditions dans lesquels elles émergent, se pérennisent et se transforment (Thelen, 2004). Les institutions génèrent en effet chez les acteurs économiques des comportements tendant à les reproduire et renforcer selon un phénomène de «cristallisation » pour aboutir à la constitution d'une "matrice institutionnelle », ensemble d'institutions propres à un territoire et une époque donnés. L'environnement institutionnel borne alors les possibilités d'adaptation d'un système en créant une "contrainte de sentier» (path dependency) pouvant aboutir à des irréversibilités, appelées effets de «verrouillage » (lock-in) : une fois formée, une institution ne peut être que difficilement supprimée et peut freiner, voire bloquer, l'adaptation du système dont elle fait partie.

Le changement institutionnel est cependant possible, qu'il soit initié par une pression extérieure au système, provienne de l'action des acteurs eux-mêmes en fonction de leurs intérêts et pouvoir d'influence, ou encore de la transformation des modèles mentaux. Selon North (1990), les institutions s'imposent aux acteurs économiques en tant que « règles du jeu », mais sont elles-mêmes un produit des comportements et stratégies desdits acteurs, ainsi que des compromis auxquels ils aboutissent dans leurs interactions. Le processus est explicité par Hamdouch, qui analyse comment les comportements des agents économiques et leurs interactions sont contraints par les institutions existantes, elles-mêmes "produit cumulatif et historique des comportements et changements passés » (Hamdouch, 2005 ; p. 247), tout en contribuant à les transformer de façon plus ou moins intentionnelle.

La perspective de Williamson, selon qui la sélection des institutions vise à maximiser l'efficience du système, est nuancée par North qui la considère comme une résultante des rapports de force entre les acteurs. Ceux-ci vont donc chercher à favoriser les institutions qui leur permettent en particulier de satisfaire leur intérêt individuel, y compris aux dépens de la performance globale du système (Didry et Vincensini, 2010). Ce changement sera graduel, les institutions informelles évoluant plus lentement que les institutions formelles, et contraint par la matrice institutionnelle d'origine. 
Un acteur essentiel du changement est l'entrepreneur institutionnel (Bourcieu, 2001), défini comme un acteur "qui crée des normes, des modèles, des valeurs et comportements en cohérence avec (son) identité et (ses) intérêts, qu'il établit comme standard et légitime vis-à-vis des autres » (DiMaggio, 1988; Zimmerman et Zeitz, 2002). La caractéristique principale de l'entrepreneur institutionnel est sa capacité à tisser de nouveaux liens répondant à la défense et la promotion de ses intérêts propres et contribuant à former une nouvelle configuration du réseau dans lequel il opère (Boyer et al., 2007).

\subsubsection{Le territoire comme construction institutionnelle}

Dans le champ des sciences sociales, le concept de territoire est avant tout relié à la science politique - champ de l'autorité publique et de la représentativité politique (Badie, 1995 ) - et tardivement intégré en géographie. Di Méo (1996), un des auteurs à y faire référence dans cette discipline, le définit comme "un espace de vie lié à des pratiques particulières, un espace vécu lié aux représentations de ces pratiques et un espace social, lié à l'imbrication des lieux et des rapports sociaux ${ }^{3} »$. On rappelle que l'analyse économique standard est a contrario fondamentalement a-territoriale, l'espace y étant essentiellement considéré comme simple support intervenant dans la décision de localisation des activités (Zimmermann, 2008). Il faut un auteur comme Marshall (1890) pour s'intéresser aux interactions entre industrie et territoire génératrices d'externalités de localisation dans son analyse des « districts industriels».

Or un domaine d'application de l'approche institutionnelle est la description, l'analyse et la comparaison des formes nationales d'économie de marché et la création de typologies de systèmes socio-économiques (modèle rhénan ou anglo-saxon, nordique, etc.), dont Albert (1991) a été le précurseur, rejoint par Hall et Soskice (2001) ou Steinmo (2010). En ce sens, elle intègre quasi-naturellement une dimension territoriale, celle du territoire national. Mais, elle peut aussi s'appliquer à des sous-ensembles de celui-ci pour offrir de nouveaux éléments de compréhension du concept de territoire.

L'économie de la proximité, courant de pensée dont certains auteurs s'y rattachent, nous offre une application pertinente de l'approche institutionnelle à la compréhension du territoire. Elle se distingue de l'économie spatiale traditionnelle en considérant des agents " situés », c'est-à-dire à la fois localisés sur un espace géographique et insérés dans un réseau de relations sociales qui conditionnent leurs actions. Elle fait une distinction entre proximité géographique, distance itinéraire traduite en temps ou en coût de franchissement, et proximité organisée (Pecqueur et Zimmermann, 2004; Rallet et Torre, 2004 ; Bouba-Olga et al., 2008).

La proximité organisée peut être appréhendée selon deux dimensions, organisationnelle et institutionnelle (Gilly et Lung, 2004). La proximité organisationnelle renvoie tout d'abord à une logique d'appartenance, qui facilite les interactions entre les agents à travers les règles et routines de comportement. Elle est un ensemble de ressources complémentaires détenues par des acteurs potentiellement aptes à participer à une même activité finalisée de type méso-économique, au sein d'une organisation (grand groupe...) ou d'un ensemble d'organisations (réseau de coopérations, secteur d'activité, système productif local).

\footnotetext{
${ }^{3}$ Voir aussi Lévy et Lussault (2003).
} 
La proximité institutionnelle traduit quant à elle une logique de similitude, à travers un système commun de représentations, de valeur et de règles, qui vont orienter la stratégie des acteurs et aboutir à des comportements de coordination réguliers (Frayssignes, 2005). Elle consiste en une "adhésion des acteurs à des règles d'action communes, explicites ou implicites (habitus), et, dans certaines situations, à un système commun de représentations, voire de valeurs, résultat de compromis provisoires entre des acteurs aux intérêts divergents et parfois contradictoires »(Gilly et Lung, 2004 ; p. 5).

Si la mise en évidence de la notion de proximité organisée est susceptible de remettre en cause la place de la proximité géographique dans la construction des interactions entre acteurs économiques, des auteurs montrent au contraire que c'est l'articulation entre les deux formes de proximité qui est à l'origine des dynamiques territoriales, la proximité géographique sans proximité organisée étant pour sa part potentiellement source de conflits (Caron et Torre, 2005).

Cette approche dépasse les limites de l'approche économique standard en intégrant le territoire, espace construit, dans l'analyse économique. Elle déjoue le biais du «territoire postulé », posé de façon exogène et sur lequel les acteurs n'ont pas prise. Le territoire se définit alors comme un espace conjuguant les différentes formes de proximité, sur lequel ont cours un ensemble d'institutions spécifiques qui contribuent à son identité.

Pecqueur (2000; p. 15) nous offre une définition du concept de territoire qui intègre cette dimension institutionnelle : il est pour lui « un espace physique borné, non pas par une juridiction ou des institutions ${ }^{4}$, mais plutôt par des éléments d'ordre naturels, culturels et/ou idéologiques. Il possède une identité qui lui est propre, déterminée par sa nature, sa culture, son histoire et son capital humain et social (connaissances, savoir-faire...), en quelque sorte par une identité collective. Il est le lieu de relations entre acteurs hétérogènes aux finalités différentes et la concrétisation d'une dynamique collective caractérisée par une finalité commune ».

Le territoire est pour Pecqueur la « constitution d'un espace abstrait de coopération entre acteurs avec un ancrage géographique, pour engendrer des ressources particulières et des solutions inédites». Cette construction s'appuie sur une proximité géographique qui circonscrit un espace particulier. Elle suppose la mise en place d'une organisation basée sur un certain degré de proximité institutionnelle, normes et valeurs partagées qu'elle contribue en retour à créer (Leloup et al. ; 2005)

\subsection{Applications aux territoires $d u$ vin}

\subsubsection{Du terroir géophysique au terroir comme territoire viti-vinicole}

La notion de territoire est centrale dans l'analyse de la filière vin en France, l'aire d'origine du vin constituant un actif spécifique, dont la mise en valeur constitue la pierre angulaire des stratégies vitivinicoles (Hinnewinkel et Le Gars, 2002). Comme le souligne Calvet (2005; p. 499), l'origine géographique du vin a acquis une telle force symbolique que même les vins les plus standardisés ne peuvent s'empêcher de l'évoquer. Toutefois, le recours à des termes variés rend la caractérisation des territoires du vin relativement complexe.

\footnotetext{
${ }^{4}$ Ici prises au sens politico-administratif du terme.
} 
Au sens strict, le terme de vignoble fait ainsi référence à une étendue de terrain plantée de vignes. Au sens large, on l'utilise pour désigner un «territoire » viticole. Le terroir est quant à lui défini dans une perspective géographique «traditionnelle » comme un « espace géographique considéré comme homogène du point de vue viticole ${ }^{5}$ ». Il est concret, tangible et cartographiable à travers de multiples facteurs - pédologie, géologie, géomorphologie, hydrologie, climatologie, microclimat, exposition - qui précèdent l'homme et existent indépendamment de lui. Les limites du terroir sont étroitement dépendantes du degré de précision avec lequel il est analysé : un découpage trop fin de l'espace géographique aboutit à sa fragmentation en une multitude de micro-parcelles, tandis qu'un degré de précision moindre ouvre la porte à des contestations entre insiders et outsiders.

L'approche institutionnelle appliquée aux territoires viticoles permet de rompre avec cette approche «naturaliste » des territoires du vin, faisant référence à un terroir exogène, immanent et immuable, pour faire ressortir leur caractère « construit », résultante des interactions entre acteurs. Rastoin et Vissac-Charles (1999; p. 173) définissent ainsi le terroir comme un «territoire homogène et doté d'une très forte identité, caractérisé par un ensemble de ressources naturelles (sol, climat) et construites (historiques, culturelles et sociales) ». Pour Polge (2003; p. 182), "le terroir n'existe pas en tant que tel, mais seulement à travers ses membres, qui le font vivre et évoluer ». Il n'est donc pas postulé, mais constitue une construction humaine, dont les composantes géographiques importent somme toute moins que les ressources induites par les acteurs et leurs interactions : savoir-faire individuels et collectifs explicites ou tacites transmis de génération en génération, ou encore confiance collective construite sur la durée réduisant les coûts de transaction et facilitant l'échange.

Le terroir est en ce sens un ensemble d'institutions ayant cours sur un espace géographique donné, qui lui donnent son identité et facilitent les interactions entre agents. Il est un système d'acteurs reliés par différents types de proximités, à savoir géographique, organisationnelle - appartenance à une industrie spécifique - et institutionnelle. Cette dernière résulte des antagonismes entre acteurs aboutissant à des compromis souvent fragiles et sans cesse renouvelés, ainsi que le montrent Laferté (2006), Jacquet (2009) ou Boyer et al. (2007) dans leur analyse des conflits autour de la délimitation de l'appellation du vin Corton en Bourgogne dans l'entre-deux-guerres.

On fera ici clairement la distinction entre le terroir géophysique, caractérisé par ses ressources naturelles, et le terroir-territoire, construction institutionnelle sociale et historique.

Les travaux de Rastoin et Vissac-Charles (1999) comme de Martin (2006) soulignent la finalité stratégique du terroir, qui repose sur un mécanisme de territorialisation, "spécification des actifs c'est-à-dire de la ressource propre au territoire qui permettra à celui-ci de se différencier vis-à-vis de son voisin plutôt que de courir après la concurrence sur des productions standards »(Pecqueur, cité par Corade et Delhomme (2008)). Pour reprendre les termes de Corade et Delhomme, celui-ci doit permettre aux acteurs de valoriser «une ressource spécifique c'est-à-dire [...] un avantage comparatif existant « naturellement » sur un territoire donné (méthode de production héritée historiquement, caractéristiques intrinsèques d'un territoire, savoir-faire $)^{6} »$ et la protéger contre la concurrence exté-

\footnotetext{
${ }^{5}$ Voir http://www.art-du-terroir.com/terroir.htm

${ }^{6}$ Nous nous écartons toutefois de cette définition dans la mesure où selon nous les avantages comparatifs évoqués peuvent être "naturels » ou « construits ».
} 
rieure. À la relation au vigneron ou négociant individuel, se substitue une relation au territoire de production du vin.

Par ailleurs, le «produit de terroir »- dans notre cas, le vin - est profondément lié à une aire géographique, mais aussi et surtout une histoire et une culture, qui lui donnent sa valeur et le rendent unique, donc inimitable. Il bénéficie en particulier d'une présomption de singularité et de qualité tenant aux compétences particulières de producteurs locaux « encastrés $^{7}$ » dans un réseau dense de relations sociales de toutes natures et construites dans la durée, qui favorise la diffusion locale des savoirs et savoir-faire. Cette identification étroite entre territoire, entreprise et produit constitue une barrière à l'entrée difficilement franchissable pour les acteurs extérieurs (outsiders), qui met les acteurs locaux (insiders) en situation de concurrence monopolistique sur les niches ainsi créées et génère une situation de « rente territoriale ».

\subsubsection{L'AOC, une institution formelle au cœur du territoire}

Le système des appellations d'origine contrôlée (AOC) constitue la pierre angulaire de la stratégie de terroir, qui permet de limiter les comportements opportunistes. Des outsiders sont en effet susceptibles de bénéficier de la rente territoriale en créant une confusion quant à la dénomination et à l'origine du produit, ainsi que le mettent en évidence les querelles portant sur l'utilisation des appellations «champagne » ou «chablis » aux États-Unis. Certaines formes concurrence entre insiders sont pour leur part susceptibles de déboucher sur l'épuisement de la rente liée au terroir (Polge, 2003).

Suivant les termes de l'INAO, une appellation d'origine d'un vin est une certification qui fait référence au "pays, région ou localité, désignant le produit qui en est originaire et dont la qualité et les caractères sont dus exclusivement ou essentiellement au milieu géographique comprenant les facteurs naturels et les facteurs humains ». La délimitation d'une appellation d'origine n'est toutefois pas plus univoque que celle des terroirs qui la composent : une seule AOC couvre ainsi l'ensemble du vignoble de Cahors, là où celui de Chablis, de dimensions proches, en compte quatre et la Bourgogne vitivinicole dans sa totalité plus de cent (Bouard et Ditter, 2008).

En tant qu'institution formelle - ensemble de normes et réglementations - l'appellation d'origine contrôlée (AOC) $)^{8}$ articule les terroirs géophysiques et le "terroir-territoire » institutionnel. Elle constitue un label ayant pour finalité de "protéger de la concurrence un produit ancré » (Torre et Filippi, 2003), en délimitant le territoire et les conditions de la production par l'imposition d'un cahier des charges contraignant.

L'AOC agit tout d'abord suivant une logique horizontale, comme un marqueur territorial garantissant à la fois l'origine et la singularité, ou typicité, du produit (Laporte, 2000). Elle agit aussi suivant une logique verticale, les contraintes de certification renforçant la présomption de qualité, dans la mesure où les produits couverts doivent présenter des caractéristiques communes indépendantes des choix individuels des producteurs. Le signal envoyé au consommateur est celui d'une « qualité différenciée » (Corade \& Delhomme, 2008). Torre (2002) ou Calvet (2005), analysent l'AOC comme un bien club offrant aux insiders, les producteurs locaux, l'accès à des externalités positives sous forme d'effets de réputation.

\footnotetext{
${ }^{7}$ Embedded, au sens de M. Granovetter (1985).

${ }^{8}$ On rappelle brièvement que les premières AOC sont nées en 1935 (décret-loi daté du 30 juillet 1935) comme une réponse aux conflits opposants vignerons et négociants sur la garantie de qualité des vins et l'appropriation de la valeur ajoutée au sein de la filière. Voir Bartoli (1990) ou Laferté (2006).
} 
L'AOC limite enfin la productivité des différents facteurs de production, donc les volumes produits. Combinée à la différenciation du produit, cette rareté voulue permet en retour de proposer des prix de vente élevés. Sa performance, à savoir sa capacité à valoriser l'origine, la typicité et la qualité du produit, repose sur la qualité de sa gouvernance, qui fait intervenir plusieurs types d'acteurs'.

Les producteurs, regroupés en un organisme de gestion et défense de l'appellation (ODG), sont collectivement les initiateurs de la démarche de certification et établissent le cahier des charges de l'AOC; ils participent par la suite à sa gestion. Le cahier des charges est validé par l'Institut National de l'Origine et de la Qualité (INAO), organisme sous tutelle de l'État, et homologué par l'État sous la forme d'un décret. L'INAO délimite aussi l'aire géographique de l'appellation et en supervise par la suite les procédures de contrôles ${ }^{10}$. L'interprofession est le lieu de rencontre et de dialogue entre l'ensemble des acteurs du vignoble (producteurs et négociants), qui contribue en particulier à la promotion et à l'amélioration de la qualité des vins.

Schéma 1 : gouvernance d'un vignoble en AOC

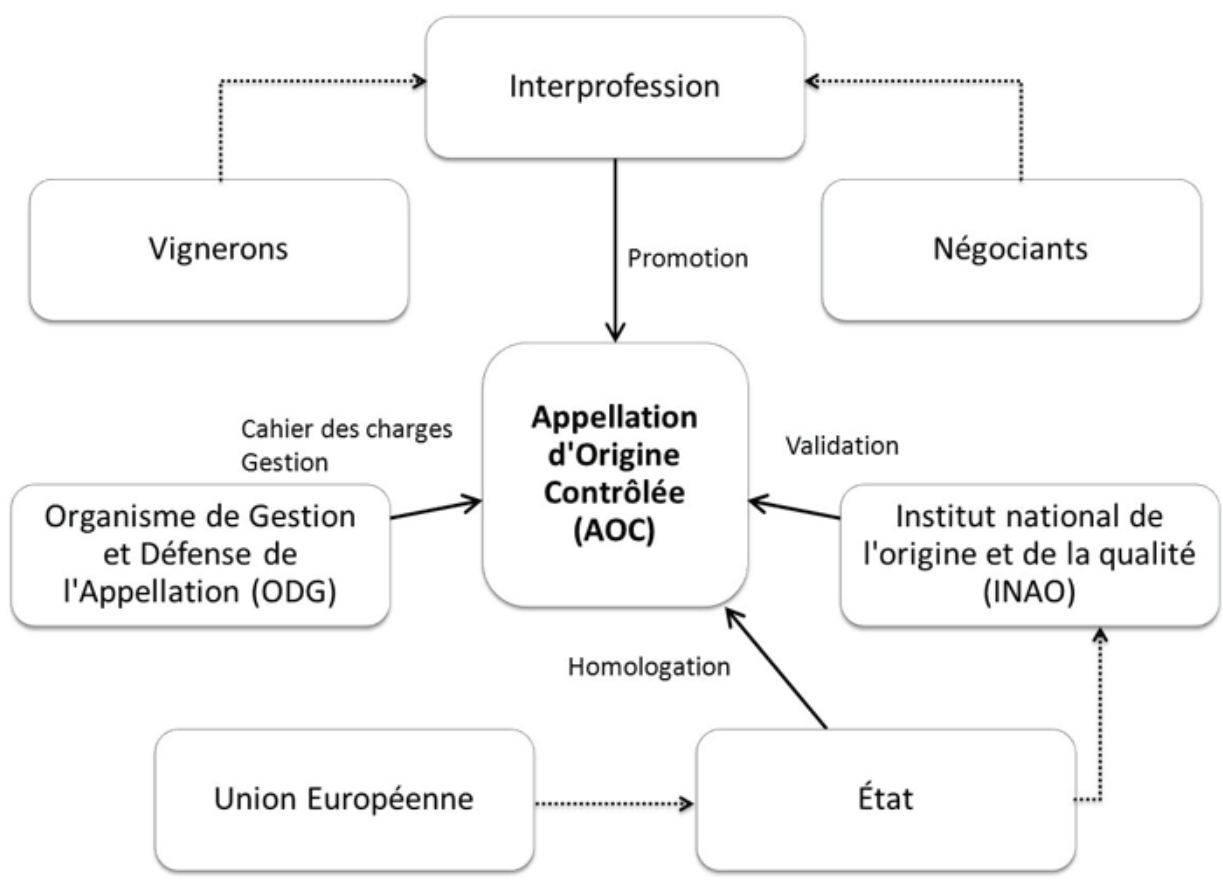

Un vignoble en AOC reste un système complexe et fragile, qui est en particulier perméable aux comportements de passager clandestin (Barrère, 2007). Certains acteurs sont susceptibles de bénéficier de la rente territoriale sans y contribuer par un effort en termes

\footnotetext{
${ }^{9}$ On ne détaille pas ici le rôle joué par l'Union européenne dans la mise en œuvre des Appellations d'Origine Protégée (AOP) au niveau communautaire.

${ }^{10}$ On distingue les autocontrôles, effectués par les producteurs eux-mêmes, les contrôles internes supervisés par l'organisme de gestion et défense de l'appellation et les contrôles externes effectués par des organismes tiers agréés par l'INAO.
} 
de qualité, avec le risque d'épuiser à terme cette dernière. Sa mise en œuvre suppose en conséquence un projet collectif, des valeurs et objectifs communs - donc un degré élevé de proximité institutionnelle - mis au service du territoire.

\section{L'AOC Cahors : une construction institutionnelle inachevée?}

\subsection{Cadrage méthodologique}

\subsubsection{Cahors, un vignoble représentatif}

À partir du cadre défini précédemment, nous avons voulu mener une recherche exploratoire visant à préciser la définition de notre problématique et suggérer des hypothèses nouvelles. La méthode des cas (Yin, 2003; Giroux, 2003), qui relate un événement ou une série d'événements (présents ou passés) pour en tirer un savoir théorique susceptible d'éclairer la pratique, nous a semblé particulièrement adaptée à une analyse longitudinale, permettant l'observation de transformations institutionnelles s'inscrivant dans le temps long (Bourcieu, 2001).

Le choix du vignoble cadurcien s'est imposé à nous pour plusieurs raisons, la première étant la simplicité de circonscription du champ de l'étude ${ }^{11}$. L'identité cadurcienne est forte (Velasco-Graciet, 2002), construite par l'histoire - ainsi l'opposition avec les vins de Bordeaux - imprégnée de traditions paysannes et de culture radical-socialiste.

Le vignoble lui-même est relativement homogène géographiquement et ses limites clairement définies; il ne comprend qu'une seule AOC, qui représente la majeure partie de la production vinicole locale. Cette AOC s'étend sur un peu moins de 4000 ha de vignes pour une aire géographique totale de 21700 ha répartie sur 45 communes le long du Lot. La production annuelle, estimée à $180000 \mathrm{hl}^{12}$, est assurée par un nombre réduit de producteurs indépendants (430 producteurs, dont 140 coopérateurs) et une seule cave coopérative, les Côtes d'Olt de Parnac ${ }^{13}$. La totalité de l'AOC est composée de vin rouge tranquille, le cépage dominant étant le malbec, qui représente $80 \%$ de l'encépagement total sur l'AOC et $11 \%$ de la superficie mondiale plantée en malbec ${ }^{14}$.

Au-delà de cette simplicité apparente, le vignoble de Cahors est aussi marqué par la complexité. Il est implanté sur deux grands types de sols, un causse calcaire kimméridgien d'une part et des sous-sols calcaires enrichis d'alluvions dans la vallée du Lot d'autre part. Une étude sur l'ensemble de l'aire géographique de l'appellation a conduit à l'identification de neuf grands terroirs géophysiques distincts, selon des critères liés à la géologie, la profondeur du sol, l'altitude et la distance par rapport au Lot, ou encore la situation de pente ou de plateau.

${ }^{11}$ Le vignoble bourguignon, dont nous sommes plus proches, est pour sa part beaucoup plus complexe, de par sa fragmentation en plusieurs sous-ensembles territoriaux (Chablis, Côtes de Beaune, Côtes de Nuits, Côte chalonnaise, Mâconnais, voire Beaujolais dans une perspective historique) et une centaine d'AOC.

${ }^{12}$ Ces données et les suivantes nous ont été fournies par l'Union interprofessionnelle des vins de Cahors (UIVC).

$1375 \%$ de la production sont actuellement assurés par des exploitants individuels et $25 \%$ par la coopérative. Depuis 2006, cette dernière fait partie de Vinovalie, regroupement de quatre caves coopératives du sud-ouest de la France.

${ }^{14}$ Pour obtenir l'AOC, un vin de Cahors doit contenir $70 \%$ de malbec au minimum. Les cépages complémentaires sont le merlot et le tannat. 
Certains de ces terroirs, essentiellement la haute terrasse dans la vallée du Lot et les terroirs du plateau calcaire du Kimméridgien, ont été identifiés comme les plus propices à la production de vins de qualité supérieure (Rouvellac, 2009), tandis que les premières terrasses sont généralement considérées comme dotées d'un potentiel moindre.

L'histoire cadurcienne est par ailleurs heurtée, ponctuée de ruptures, avec la quasidisparition de la vigne entre la fin du $\mathrm{XIX}^{\mathrm{e}}$ et le milieu du XX $\mathrm{XX}^{\mathrm{e}}$ siècle, sa renaissance dans les années 1960 et la création de nouvelles institutions, sans oublier la crise actuelle, elle aussi porteuse de changements. Cahors représente en ce sens un microcosme de la filière vitivinicole française et un laboratoire des transformations en cours.

Pour mener à bien cette recherche, nous avons fait appel à diverses sources. La littérature académique nous a fourni notre grille d'analyse. Les données factuelles proviennent quant à elles directement des acteurs concernés, au premier rang desquels l'Union interprofessionnelle des Vins de cahors (UIVC), mais aussi de la presse quotidienne régionale. Ces données ont été complétées par des dires d'expert.

Durant le printemps 2009, un premier questionnaire a été administré à vingt-sept acteurs de la filière locale et différents entretiens conduits sur la base d'un guide prédéfini. Des visites ont été menées dans six domaines représentatifs. Un second questionnaire plus détaillé a été envoyé par courrier à 210 producteurs de l'appellation en décembre 2009, dont 35 ont répondu. Notre dernière source a été une étude menée par les étudiants de l'École Supérieure Agronomique de Toulouse (ENSAT), dirigée par Valérie Olivier (2009), dont nous avons pu reprendre certaines conclusions.

\subsubsection{Une histoire longue et difficile}

Le Cahors est connu pour être l'un des plus anciens vins d'Europe, ayant été implanté par les Romains dès 50 avant J.C. En 1152, le mariage d'Aliénor d'Aquitaine avec Henri Plantagenêt, futur roi d'Angleterre, impulse son développement rapide et les exportations du Black Wine atteignent $850000 \mathrm{hl}$ au début du XIV siècle, soit la moitié des exportations du port de Bordeaux. La guerre de Cent Ans met fin à cette première période de prospérité : à partir de 1373, les obstacles placés par les négociants bordelais à la commercialisation du Cahors étouffent peu à peu la production locale.

Après une reprise du XVIII ${ }^{\mathrm{e}}$ à la première partie du $\mathrm{XIX}^{\mathrm{e}}$ siècle - le vignoble s'étend alors sur 80000 ha et $75 \%$ de la production sont exportés - le phylloxéra entraîne dès 1876 une nouvelle baisse de la production, pouvant dans certains cas atteindre $90 \%$. Elle ne reprend qu' au début du $\mathrm{XX}^{\mathrm{e}}$ siècle, à un niveau très inférieur à celui de la période précédente : le vignoble ne représente alors plus que 25000 ha, occupés par des cépages peu qualitatifs à rendement élevé.

À la fin de la Seconde Guerre Mondiale, seuls subsistent quelques producteurs, généralement des polyculteurs ${ }^{15}$, qui vont durement subir les gelées de 1956. Mais, c'est à cette époque que sont prises les mesures qui permettront de relancer le vignoble. La cave coopérative des Côtes d'Olt est créée en 1947 par 146 viticulteurs locaux désireux de relancer la culture du cépage traditionnel local, le Malbec. En

\footnotetext{
${ }^{15}$ Rappelons que Jean Fourastié (1979) avait mené une analyse restée fameuse de l'évolution de l'un des villages du vignoble, Douelle, entre 1946 et 1976. Le lecteur intéressé pourra en particulier y trouver une description de la situation du vignoble à la fin de la $2^{\text {nde }}$ Guerre Mondiale.
} 
1951 le vin de Cahors obtient le label VDQS (vin délimité de qualité supérieure). En 1971, suite à l'adoption d'une AOC Cahors donnant une place centrale au cépage Malbec, sa réputation va devenir nationale, voire internationale. Le vignoble connaît alors un développement fulgurant : sa surface, 440 ha lors de son accession à l'AOC, a été multipliée par dix en 2001.

Il rentre de nouveau en crise au début du XXI ${ }^{\mathrm{e}}$ siècle : celle-ci éclate en décembre 2002, lorsque la présentation d'un projet de hiérarchisation de l'AOC est contestée par une partie des petits exploitants, selon lesquels celui-ci aboutirait à déclasser de l'appellation une partie de leurs parcelles les moins qualitatives, situées dans les premières terrasses. Le conflit qui en résulte est probablement l'un des plus violents qu'ait connu le vignoble français contemporain.

L'AOC Cahors connaît jusqu'en 2008 une situation difficile, marquée par une baisse marquée des grands indicateurs de valorisation que constituent le prix des vignes, le cours du vrac, le revenu moyen à l'hectare et le $\mathrm{ROI}^{16}$ (cf. schéma 2). Ses vins connaissent un problème sérieux de débouchés - 80 à $85 \%$ des volumes étant vendus en France, principalement par le biais de la grande distribution - et de valorisation, $45 \%$ des vins vendus en hypermarchés l'étant à moins de 2 euros $^{17}$. L'offre est en outre structurellement supérieure à la demande, entraînant l'augmentation régulière des stocks (schéma 2). Il s'ensuit une gestion malthusienne du vignoble, caractérisée par la baisse continue de la surface revendiquée (schéma 3 ) et du nombre de producteurs (630 exploitations en 1986, 430 en 2010).

Schéma 2 : l'AOC Cahors, prix et revenus

Données UIVC

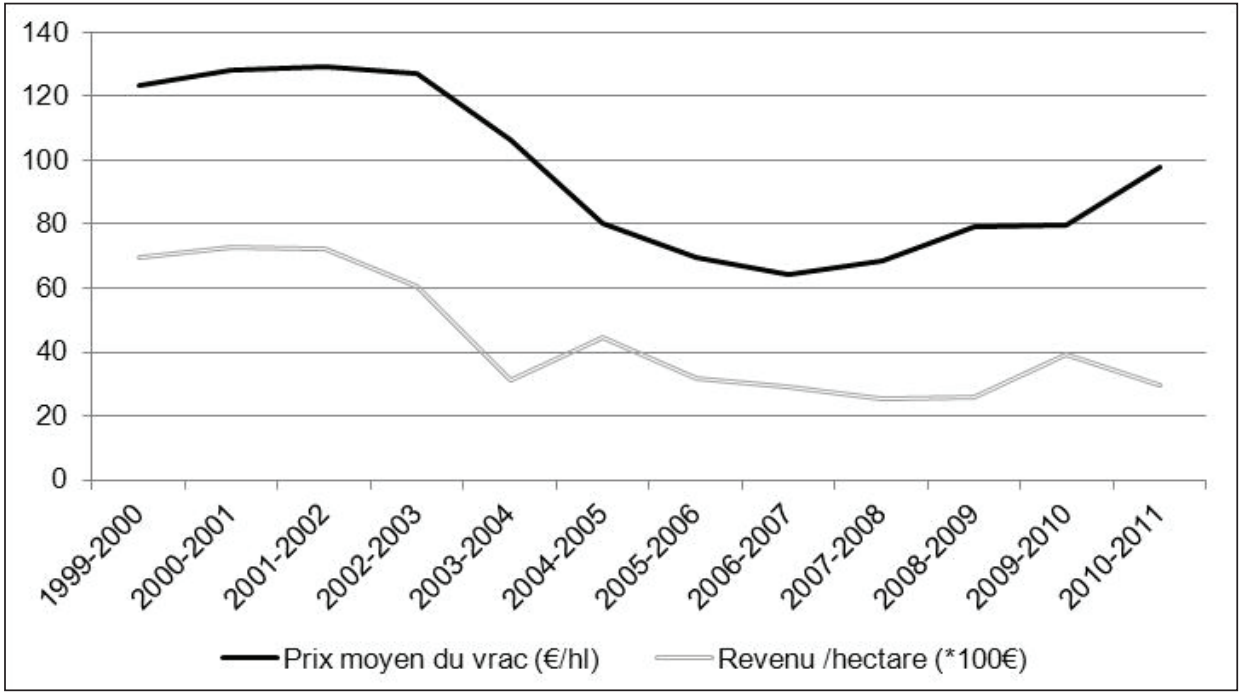

\footnotetext{
${ }^{16}$ Rapport bénéfice/investissement.

${ }^{17}$ Données UIVC.
} 
Schéma 3 : l'AOC Cahors, surface et stocks

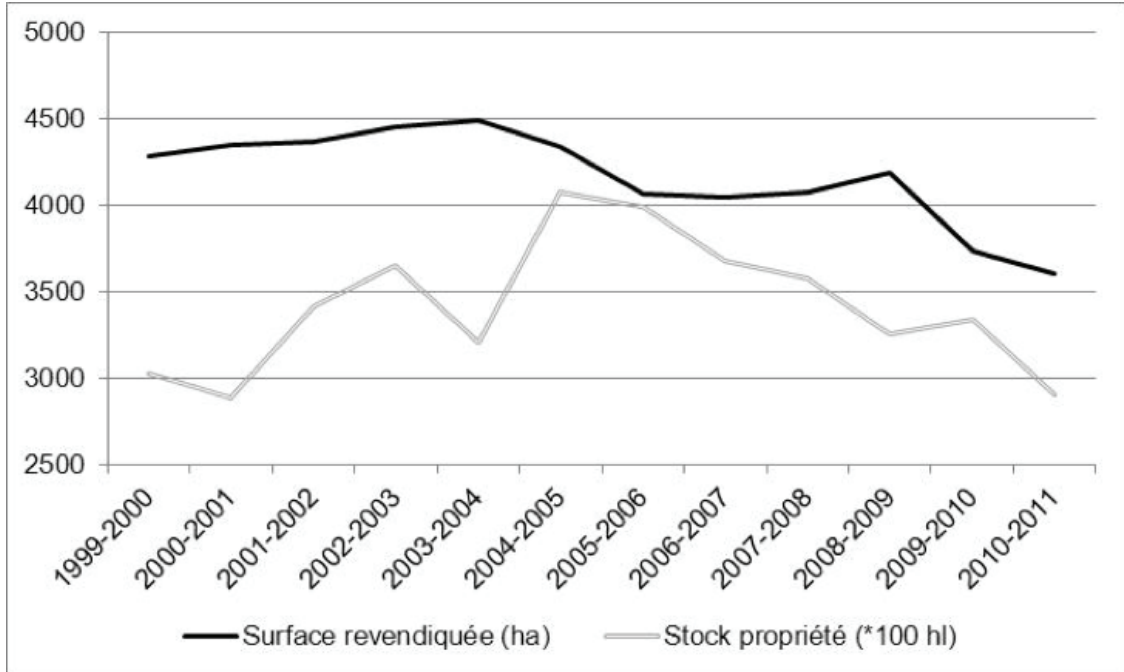

Données UIVC

\subsection{Un territoire inachevé}

\subsubsection{Une matrice institutionnelle marquée par les ruptures et divergences}

Le cœur des difficultés récemment rencontrées par le vignoble de Cahors tient selon nous aux faiblesses de l'AOC en tant qu'institution formelle articulant terroir géophysique et terroir-territoire, elles-mêmes reflet d'une construction institutionnelle inachevée.

Dès les années 1980, l'absence de différenciation au sein de l'AOC Cahors est identifiée par les professionnels comme une des grandes faiblesses du vignoble : l'AOC unique ne remplit pas son rôle de façon satisfaisante car elle ne permet pas d'effectuer de distinction horizontale entre les différents terroirs géophysiques ni de distinction verticale entre vins de qualités différentes (c.f. 1.2.2.). Il en résulte une incertitude marquée pour le consommateur, portant à la fois sur la typicité et la présomption de qualité du produit, seule l'origine étant garantie par l'AOC. Cette situation tend à favoriser des pratiques de free riding - les producteurs les moins qualitatifs bénéficiant de l'appellation sans y contribuer - qui tirent l'ensemble du vignoble vers le bas et dégradent sa réputation globale. Ce diagnostic, connu de longue date et relativement partagé, ne se traduit pourtant pas par un consensus quant aux remèdes à mettre en œuvre. Tout au contraire, plusieurs tentatives de réforme de l'AOC Cahors vont échouer. Le pilotage efficace d'une $\mathrm{AOC}$, institution formelle, suppose en effet des règles communes d'action et de gestion des conflits, une convergence de vues et valeurs, qui sont difficiles à identifier dans le cas cadurcien. L'environnement institutionnel y apparaît au contraire dominé par des tensions entre individuel et collectif, entre hiérarchie et égalitarisme.

Le questionnaire administré en 2008 montre ainsi que les acteurs locaux considèrent en majorité que leurs principaux concurrents ne sont pas tant les producteurs d'autres régions françaises ou internationaux, que les autres producteurs locaux, leurs voisins (schéma 4). Les relations au sein du vignoble sont pour leur part considérées comme essentiellement marquées par l'individualisme, la méfiance, la jalousie et la concurrence (schéma 5). 
Schéma 4 : Réponses à la question : « qui sont vos principaux concurrents? » (réponses de rang 1)

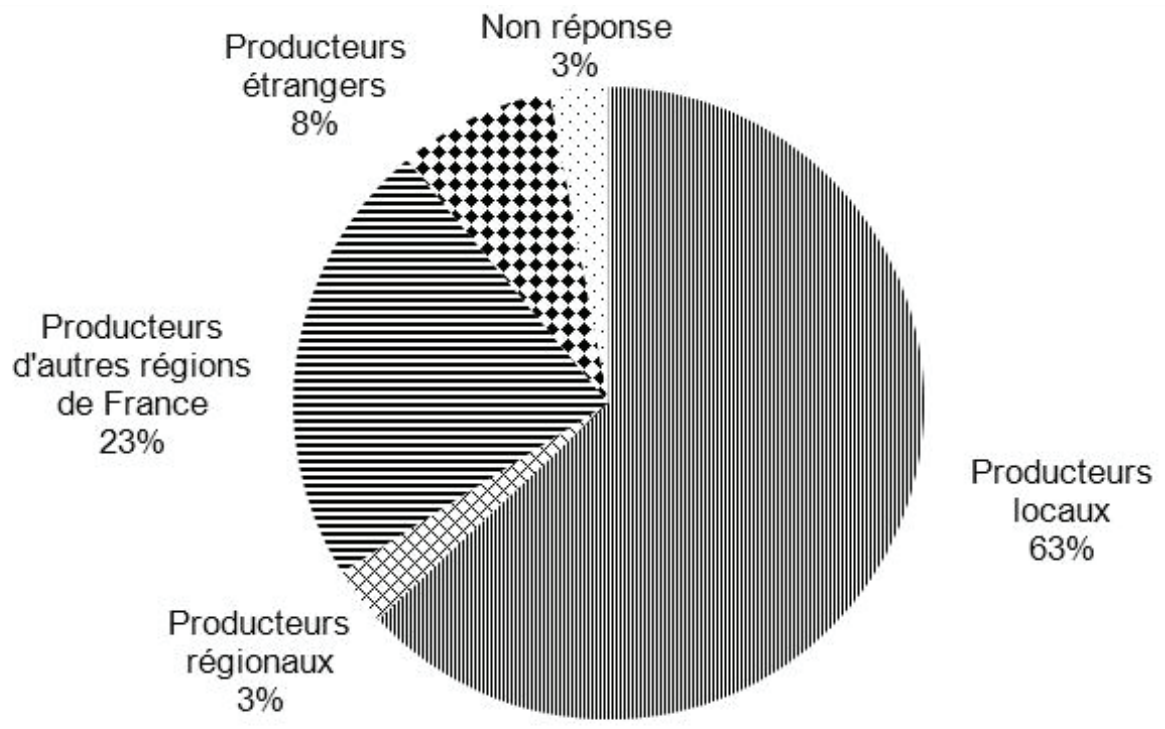

Schéma 5 : Perception de la nature des relations entre producteurs de l'AOC (réponses de rang 1)

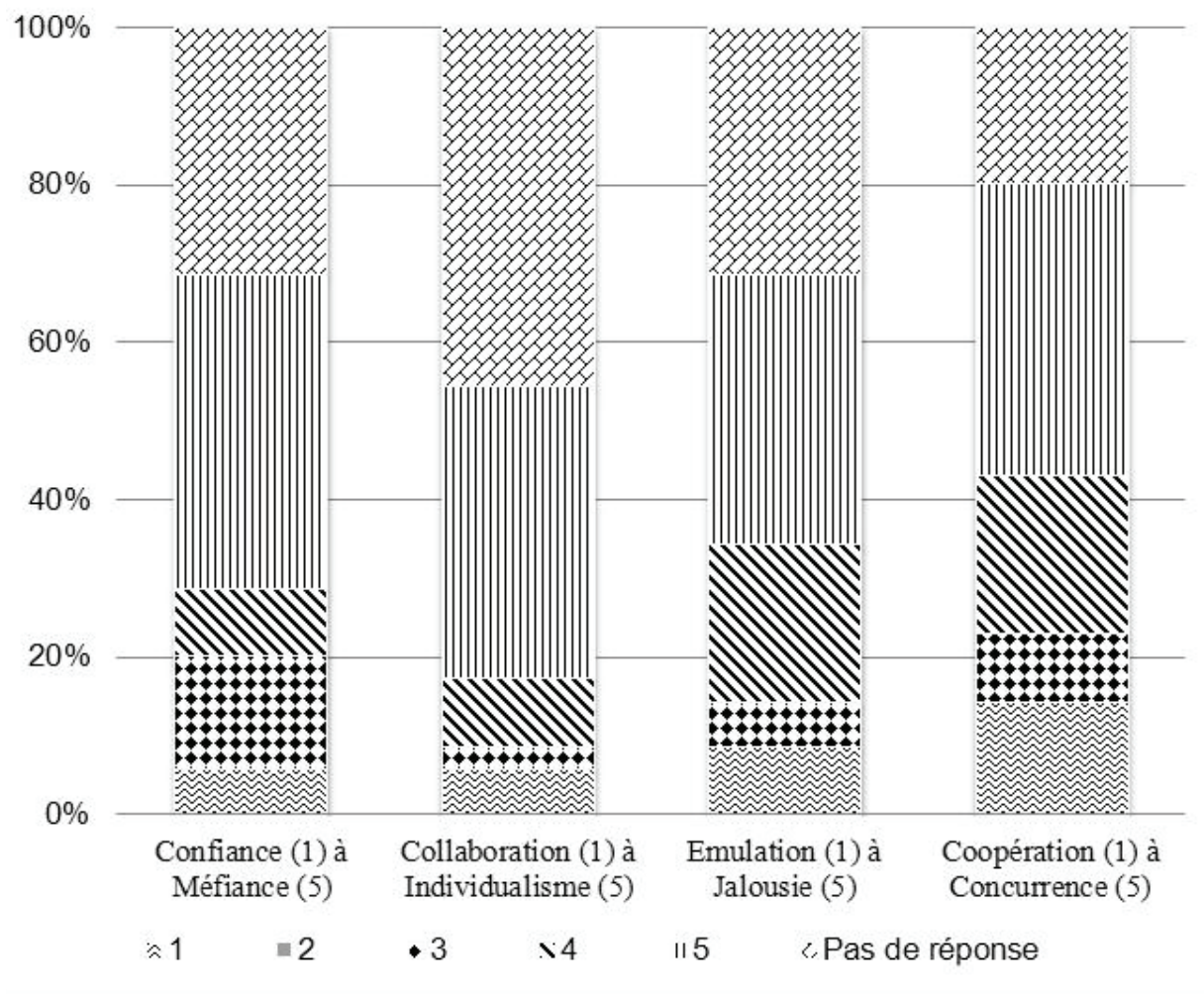


Les lignes de fracture et axes de confrontation entre acteurs du vignoble se sont en effet multipliés, pesant sur sa gestion et sa performance. Des mutations répétées dès avant l'adoption de l'AOC expliquent en partie cette situation en mettant en relief l'affaiblissement de sa matrice institutionnelle d'origine et la superposition continue de strates institutionnelles parfois incohérentes.

La crise phylloxérique a constitué la première de ces mutations (Velasco-Graciet et Rouvellac, 2002) : elle a entraîné la quasi-disparition du vignoble au XIX ${ }^{\mathrm{e}}$ siècle et en conséquence celle d'un ensemble de pratiques et techniques, de "tours-de-main », mais aussi de valeurs qui lui étaient liées. Les seuls producteurs qui subsistent encore dans les années 1950 sont avant tout des agriculteurs, voire des paysans, qui voient dans la production viticole une activité et une source de revenus parmi d'autres.

Un mouvement de transformation est porté à partir des années 1960 par la cave coopérative : elle va agir en tant qu'entrepreneur institutionnel pour promouvoir de nouveaux comportements et normes axés sur la recherche de qualité et de typicité, qui s'imposeront à une partie des producteurs. Dans les années 1960, il est ainsi décidé à l'initiative de son président de privilégier la qualité de la production par la réduction des rendements et le choix de cépages sélectionnés assurant une certaine typicité. La cave coopérative va aussi pousser au développement de la vente en bouteille, plus rentable que celle du vin en vrac, qui lui permet de verser à ses adhérents un prix double de celui du vin de consommation courante et de compenser ainsi les efforts fournis en termes de rendements et d'investissement (Tulet et Velasco-Graciet, 2003).

Ces choix stratégiques vont impulser le déploiement qualitatif et quantitatif de la production, la coopérative jouant un rôle de premier plan jusqu'au début des années 1970 : elle vinifie à cette époque plus de la moitié de la production locale (Tulet et VelascoGraciet, 2003). Une partie des producteurs vont quant à eux marquer leur identité en se regroupant en une Confrérie du Vin de Cahors, créée en 1964. Ces comportements et normes sont formalisés et valorisés par l'adoption de l'AOC Cahors ${ }^{18}$, qui fait intervenir des réseaux politiques locaux et nationaux : le décret d'adoption est signé en 1971 par le Président G. Pompidou, lui-même connu pour être un amateur de vin de Cahors.

L'action de la coopérative va favoriser le développement rapide de la production, mais aussi la dualisation du vignoble. Un premier sous-groupe est composé de viticulteurs dont l'activité est marquée par une polyculture traditionnelle. Ils sont établis de longue date ou ont cherché à bénéficier de la notoriété croissante du vin de Cahors à partir des années 1970. Leur exploitation est généralement de petite taille, située dans les premières terrasses et ils sont dotés d'un bagage technique limité. Disposant de volumes réduits qu'ils écoulent à bas prix, ils se sentent peu concernés par les problématiques de qualité et de montée en gamme. Ils estiment bénéficier de l'image de l'AOC unique qui a permis l'extension rapide du vignoble et de la production dans les années 1970 à 1990 et craignent un déclassement en cas de hiérarchisation de l'AOC, à laquelle ils s'opposent.

Si ces petites exploitations polyculturales dominent jusque dans les années 1970, la vigne paysanne est par la suite progressivement supplantée par ces professionnels de la viticulture (Tulet et Velasco-Graciet, 2003). Un second sous-groupe est en effet composé d'une nouvelle

\footnotetext{
${ }^{18}$ Son cahier son cahier des charges est à cette époque considéré comme l'un des plus stricts au niveau national (Olivier, 2009).
} 
génération de vignerons indépendants exploitant des domaines de taille croissante, grâce à qui l'AOC se modernise et se développe. Pour partie les descendants d'une première génération de viticulteurs ou anciens coopérateurs, ces vignerons spécialisés sont parfois aussi des outsiders, des nouveaux venus dotés de moyens financiers importants, qui se spécialisent sur la production haut de gamme. Ils disposent d'un bagage technique supérieur à la catégorie précédente qui leur permet de mettre en œuvre un processus d'innovation constante.

Souhaitant améliorer la valorisation de leurs vins, les nouveaux producteurs soutiennent le processus de hiérarchisation. Ils vont chercher à agir en tant qu'entrepreneurs institutionnels et chercher à légitimer et imposer leurs normes. Certains d'entre eux se sont regroupés au sein d'une association des Seigneurs-Cahors pour définir en 1999 une charte de qualité basée sur la maîtrise des rendements, présentée comme "l'école des crus » (Velasco-Graciet et Rouvellac, 2002). Perçue comme contraignante, élitiste et fermée, ne tenant pas compte des intérêts de la masse des producteurs, elle dépérit progressivement pour ne plus comprendre actuellement que 25 producteurs environ, qui n'assurent que des volumes insuffisants pour relancer une dynamique collective.

Une deuxième ligne de partage divise les vignerons indépendants et les coopérateurs : les seconds sont suspectés par les premiers de favoriser la concurrence par les prix en vendant leur raisin à bas prix à la cave coopérative. Celle-ci, dont l'importance a commencé à décliner à partir des années 1970 (cf. 2.1.2.), s'est détournée de sa stratégie initiale de promotion de la qualité pour rechercher la massification des ventes dans l'entrée de gamme en grande distribution et le «hard discount », où elle est suivie par certains opérateurs, contribuant à affaiblir l'image de l'appellation d'origine ${ }^{19}$. Les antagonismes entre exploitants s'exacerbent alors.

Cette double confrontation aboutit en décembre 2002 au refus du projet de hiérarchisation du vignoble évoqué précédemment (cf. 2.1.2.), les petits producteurs contestant une réforme trop brutale à leurs yeux et craignant de perdre le droit à l'appellation. Elle crée une situation de blocage, dans laquelle aucun acteur n'est plus à même de légitimer ses normes sur l'ensemble du vignoble.

En conséquence, le seul choix offert aux producteurs spécialisés engagés dans une stratégie de qualité et prix élevés est de rechercher des modes de valorisation individuels plutôt que collectifs, la réputation propre du vigneron ou du négociant l'emportant sur l'image collective véhiculée par l'AOC. Il en résulte une hiérarchisation de facto, mais aussi une multiplication des marques et mentions individuelles (clos, domaines, châteaux) qui contribue à son tour à brouiller l'image de l'appellation, sans que ne puisse se mettre en place ce que Laferté (2006) appelle un modèle « aristocratique » de type bordelais, qui nécessiterait un classement individualisé officiel des exploitations.

Le statu quo ne profite pas non plus aux petits producteurs, qui ne sont pas à même de s'opposer à la pression des négociants dans un contexte de dévalorisation de l'image du vignoble. Ils se voient imposer des prix de vente proches, voire inférieurs, à leurs coûts de production, face auxquels ils ne peuvent perdurer qu'en épuisant progressivement le capital accumulé durant les années fastes.

Le groupe des négociants est en effet lui aussi partagé : les vignerons-négociants locaux dont une priorité était d'assurer un débouché à leur production propre ont peu à peu été remplacés ou rachetés par des opérateurs extérieurs dotés d'un pouvoir de marché sen-

${ }^{19}$ La cuvée « carte noire », peu qualitative et vendue à bas prix, en sera le symbole le plus marquant. 
siblement plus important et dont l'objectif premier est d'élargir leur approvisionnement. Faiblement impliqués dans un vignoble dont ils estiment l'image dégradée et la qualité insuffisante, ils ont imposé des prix d'achat peu élevés, qui pèsent sur l'ensemble des opérateurs. Certains vignerons chercheront à se substituer au négoce en commercialisant leurs vins eux-mêmes, sans toutefois nécessairement posséder les compétences requises ni pouvoir s'affirmer en tant qu'opérateurs de premier plan (Olivier, 2009).

Ces différentes lignes de fracture se répercutent à leur tour sur les acteurs dits «institutionnels ». La crise de décembre 2002 va ainsi entraîner la scission temporaire du syndicat des producteurs, résolue depuis, tandis qu'une partie des acteurs moteurs du vignoble se désintéressait de l'interprofession.

Schéma 6 : Acteurs et gouvernances du vignoble cadurcien

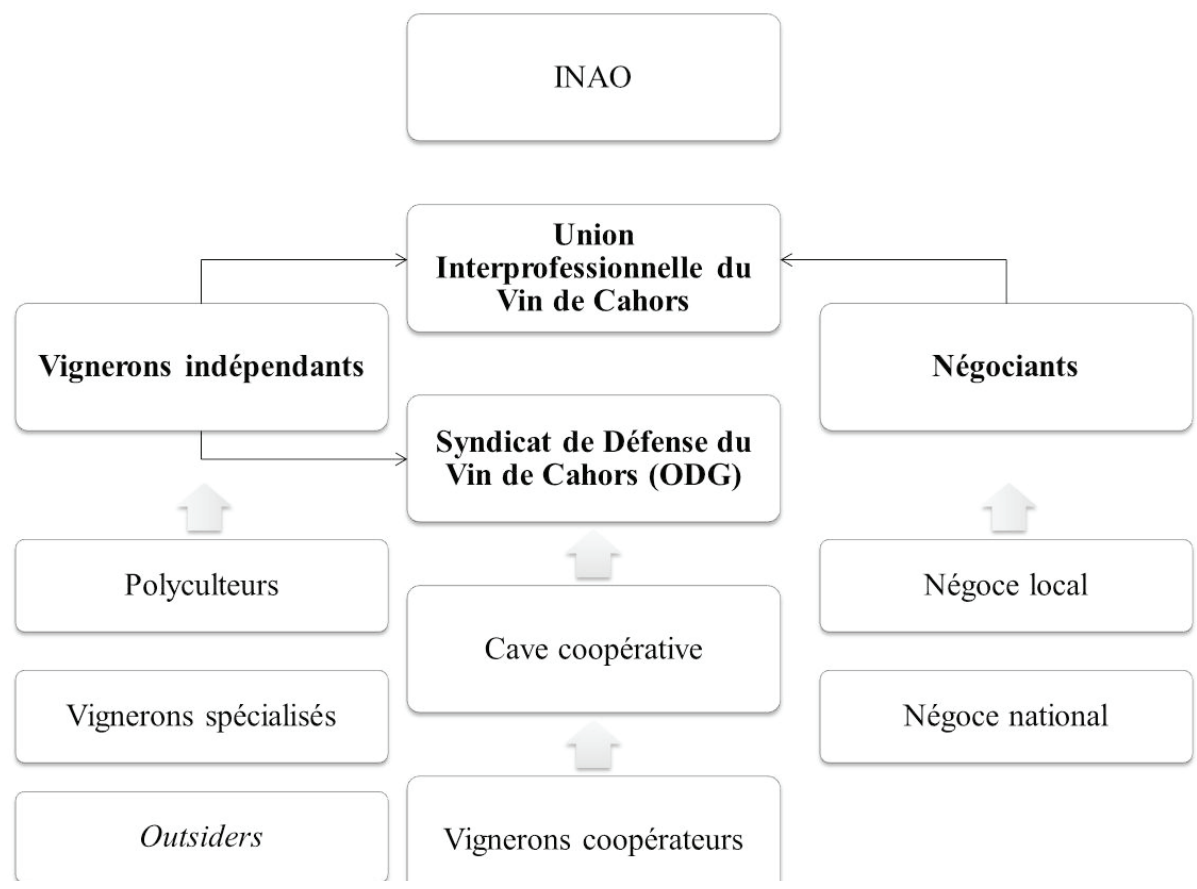

\subsubsection{Une recomposition en cours}

La situation du vignoble est mouvante, les différents groupes précédemment étudiés travaillant à sa reconfiguration avec pour enjeu l'achèvement d'un véritable territoire au sens institutionnel du terme, support de l'identité vitivinicole locale. La perception de l'urgence de la situation ayant eu raison des réticences, une nouvelle tentative de réforme a été lancée en 2008, fondée sur un "Plan Malbec » visant à repositionner l'offre de Cahors dans le cadre d'une gamme plus large et plus vendeuse, notamment sur les marchés étrangers.

Ce plan a pour objet de capitaliser sur l'engouement du cépage malbec, originaire du Lot et mis en valeur par les producteurs argentins, pour pénétrer les marchés internatio- 
naux, en particulier nord-américains, en proposant une segmentation nouvelle articulant AOC et indication de cépage ${ }^{20}$. L'AOC Cahors reste la pierre angulaire du vignoble au niveau national, mais est complétée au niveau international par une référence au malbec, principal cépage entrant dans la composition des vins de Cahors en AOC.

Cette forte identification AOC Cahors/cépage malbec doit permettre de redéfinir l'identité du vignoble cadurcien en contournant les fractures actuelles pour fédérer les acteurs autour d'une nouvelle segmentation de l'offre (tableau 1), suivant une logique verticale proche de celle mise en œuvre dans les années 1960 à Chablis (Brouard et Ditter, 2008). Elle facilite la diversification de la production et la hiérarchisation de facto de l'appellation au niveau international, sans être perçue comme une source de déclassement par les petits exploitants. Parallèlement, une réforme de l'agrément et la modification du cahier des charges de l'AOC ont été entamées, tandis que la production et les ventes de Vins de pays du Lot traditionnellement minoritaires se développaient pour les vins d'entrée de gamme.

Tableau 1 : une nouvelle segmentation du marché

\begin{tabular}{|l|l|l|l|l|l|l|}
\hline $\begin{array}{l}\text { Segment } \\
\text { de marché }\end{array}$ & Label & Caractéristiques & $\begin{array}{l}\text { Marchés } \\
\text { potentiels }\end{array}$ & $\begin{array}{l}\text { Situation } \\
\text { en 2011 } \\
\text { (\%) du } \\
\text { volume } \\
\text { total) }\end{array}$ & $\begin{array}{l}\text { Objectif } \\
\text { pour 2015 } \\
\text { \%o du } \\
\text { volume } \\
\text { total) }\end{array}$ & $\begin{array}{l}\text { Prix } \\
\text { départ } \\
\text { cave }\end{array}$ \\
\hline Icon & $\begin{array}{l}\text { Cahors } \\
\text { Grand } \\
\text { Cru }\end{array}$ & AOC renforcée & $\begin{array}{l}\text { Français et } \\
\text { international }\end{array}$ & $8 \%$ & & $+14 €$ \\
\hline $\begin{array}{l}\text { Super } \\
\text { Premium }\end{array}$ & $\begin{array}{l}\text { Cahors- } \\
\text { Malbec }\end{array}$ & $\begin{array}{l}\text { AOC }+85 \% \\
\text { Malbec }\end{array}$ & International & $42 \%$ & & 7 à $147 €$ \\
\hline Premium & $\begin{array}{l}\text { Cahors } \\
\text { AOC }\end{array}$ & $\begin{array}{l}\text { Français et } \\
\text { international }\end{array}$ & $20 \%$ & 5 à $7 €$ \\
\hline $\begin{array}{l}\text { Popular } \\
\text { Premium }\end{array}$ & $\begin{array}{l}\text { Vin } \\
\text { de Pays } \\
\text { du Lot }\end{array}$ & Vin de pays & Français & $30 \%$ & $30 \%$ & 3 à $5 €$ \\
\hline
\end{tabular}

Données IUVC

Cette nouvelle réforme coïncide avec le renouvellement progressif du tissu productif, qui laisse une place croissante à des exploitants et négociants plus jeunes et/ou non originaires du vignoble susceptibles de contribuer au renouvellement et à la montée en gamme de l'offre. Au-delà du succès rencontré par les malbecs argentins aux États-Unis, cette stratégie doit donc faciliter la mise en œuvre de nouvelles règles du jeu, dépassant les conflits contemporains et réunissant les professionnels du vin de Cahors autour d'un projet commun de développement de la filière. Ce projet n'est d'ailleurs pas porté par un producteur ou négociant du vignoble s'imposant en tant qu'entrepreneur institutionnel, mais bien par l'interprofession, organisme représentant l'intérêt commun.

Ce «Plan Malbec » semble à l'heure actuelle être couronné de succès dans la mesure où la performance du vignoble et de ses vins a sensiblement été améliorée entre 2008 et 2011 (sché-

${ }^{20}$ Voir « Réussir Vigne » $\mathrm{n}^{\circ} 153$, juin 2009. 
mas 2 et 3) : la valorisation moyenne du vrac a par exemple fortement augmenté, dépassant de nouveau $100 € / \mathrm{hl}$ après plusieurs années de baisse. L'ouverture internationale s'est elle aussi accrue : la valeur des exportations s'élevait à 1,6 Mn € (HT) en 2010, contre $1 \mathrm{Mn} €$ en 2008 alors que le prix moyen de vente à l'export passait de 2,96 €/l en 2008 - en quasi-stagnation depuis 2002 - à 4,45 €/l sur les huit premiers mois de 2011 (données IUVC).

Mais, ces succès récents et l'enthousiasme qu'ils suscitent ne doivent pas occulter les obstacles persistants que peut rencontrer cette stratégie sur le moyen terme. L'amélioration de l'image et le développement des exportations sont encore portés à l'heure actuelle par un petit nombre d'opérateurs, essentiellement des vignerons spécialisés (cf. supra 2.2.1. Une matrice institutionnelle marquée par les ruptures et divergences). Or, à la croissance espérée de la demande en malbec de Cahors doit pouvoir répondre une croissance de l'offre, tant quantitative que qualitative. Une pénurie relative de l'offre, tout comme la mise en marché de vins peu qualitatifs, détournerait par ailleurs les consommateurs de vins de Cahors vers d'autres produits. Il existe donc un risque que seule une élite des producteurs ne bénéficie de l'embellie, la crise persistant chez la majorité d'entre eux, qui rejetteraient dès lors la transformation en cours.

Les choix de l'UIVC ne sont d'ailleurs pas acceptés par tous les acteurs du vignoble. L'identification croissante entre AOC Cahors et cépage malbec est ainsi critiquée en raison d'un appauvrissement possible de l'identité locale rendant les vins de Cahors fortement dépendants d'effets de mode éphémères aux États-Unis en faveur d'un cépage ou d'un autre. On lui oppose aussi un risque de « cannibalisation » entre vins en AOC et vins de pays faisant appel au même cépage, ces derniers bénéficiant de conditions de production et de rendement nettement moins contraignantes ${ }^{21}$.

Pour finir, la décision de l'UIVC de ne pas rejoindre la nouvelle interprofession des Vins du Sud-Ouest (IVSO) afin de préserver l'identité cadurcienne a elle aussi été contestée. Ses détracteurs jugent qu'elle prive les producteurs locaux de subventions, d'une opportunité de mutualisation des coûts de promotion et commercialisation, voire de la visibilité d'une interprofession régionale.

En dépit de ses premières réussites, il est donc encore trop tôt pour conclure que le «Plan Malbec » a correctement joué son rôle de catalyseur du changement institutionnel sur le vignoble de Cahors. Il a toutefois clairement participé à la mise en place d'une dynamique positive et facilité la réduction de certaines divergences entre acteurs locaux.

\section{Conclusion}

Sous la pression de la concurrence internationale et de la contraction de son marché intérieur, la filière vitivinicole française est actuellement entrée dans une phase de «bouillonnement créatif », ses acteurs cherchant à s'adapter à leur nouvel environnement par la transformation du modèle traditionnel et/ou la structuration progressive de nouveaux modes d'organisation, de production et de valorisation des vins.

À partir d'un cas précis, nous avons voulu dans cet article exposer comment le recours à l'approche institutionnelle permet d'éclairer la situation d'un vignoble français en situa-

\footnotetext{
${ }^{21}$ Les rendements maximums sont par exemple de 90 hectolitres/hectare pour les vins de pays (ou indications géographiques protégées (IGP)) contre 40 hectolitres/hectare pour les AOC.
} 
tion de crise ou mutation. On montre en particulier que la référence partagée au terroir tend à camoufler les stratégies des différents acteurs du vignoble et leurs divergences, qui sont essentielles à la bonne compréhension de ses forces, faiblesses et de possibles dépendances de sentier.

Dans un tel contexte, l'AOC apparaît comme un socle institutionnel commun a minima pour l'ensemble des producteurs du vignoble, qui ne suffit toutefois pas à assurer une performance économique satisfaisante en l'absence d'un degré suffisant de proximité institutionnelle. Par ailleurs, l'absence d'un acteur susceptible d'agir en tant qu'entrepreneur institutionnel pour légitimer ses normes auprès de l'ensemble des autres acteurs du vignoble peut empêcher la remise en cause du statu quo ante, quand bien même celui-ci se révèle être à moyen terme porteur d'un jeu à somme négative.

Cahors représente pour nous un exemple à la fois simple et représentatif de la situation des vignobles français en AOC, aux contours bien déterminés et marqués à la fois par l'histoire et la géographie. L'analyse est toutefois transposable à d'autres contextes. Laferté (2006), Jacquet (2009) ou Boyer et al. (2007) ont montré comment les transformations du vignoble bourguignon dans les années 1930 ont été portées par des entrepreneurs institutionnels mobilisant des réseaux de natures diverses dans les champs politique, économique et culturel pour imposer l'idée que l'origine de la vigne doit être son premier critère de la qualité, idée qui sera validée par la mise en place progressive des appellations d'origine contrôlée ${ }^{22}$. Toujours en Bourgogne, la renaissance du vignoble chablisien dans les années 1960 et son succès inattendu jusqu'à la crise récente ont été la résultante d'une stratégie collective portée par des acteurs locaux partageant des normes et valeurs communes, qui ont permis une gestion dynamique des quatre $\mathrm{AOC}$ du vignoble (Ditter et Brouard, 2008).

Barrère (2007) s'est quant à lui intéressé au cas de la Champagne, où les grandes maisons ont su tirer profit de la mise en place des AOC pour imposer à l'ensemble du secteur une politique de qualité et à l'aide d'un processus de lobbying auprès des pouvoirs publics et de compromis entre les différents types d'acteurs pour éliminer les stratégies alternatives.

Ces différents exemples mettent en évidence l'importance de la composante institutionnelle dans la performance des vignobles considérés, tout comme la diversité des trajectoires possibles, chaque vignoble composant un environnement institutionnel spécifique, fonction de la géographie et de l'histoire. Si chaque vignoble est unique, l'expérience de l'un peut en revanche éclairer la situation d'un autre, faciliter la définition et la mise en œuvre d'une stratégie d'adaptation.

${ }^{22}$ Une synthèse de ces travaux est disponible dans Bourcieu et al. (2010). 


\section{Références}

Albert M., 1991. Capitalisme contre capitalisme. Le Seuil, Paris.

Badie B., 1995, La Fin des territoires. Fayard, Paris.

Barrère C., 2007. Un modèle évolutionnaire de segmentation des marchés du vin. Politiques de qualité et caractéristiques informationnelles. Revue d'Économie Rurale, septembre-octobre.

Barrère C., Ditter JG, Brouard J., 2010. La compétitivité des modèles de terroir dans le contexte de la globalisation des marchés du vin. XLVII Colloque de l'ASRDLF, Aoste, 20-22 septembre.

Bartoli P., Boulet D., 1990, Régulation et spécificités sectorielles : la sphère viticole. Cahiers d'Économie et de Sociologie Rurales 17, 7-38.

Bouba-Olga O, Carrincazeaux C., Coris M., Eds 2008. La Proximité : 15 ans déjà ! Revue d'Économie Régionale et Urbaine 3.

Bourcieu S., 2001. PMI exportatrices et transformations de la stratégie dans les pays en transition. Revue Internationale PME 14 (1), 69-92.

Bourcieu S., Ditter JG, Brouard J., 2010. Les entrepreneurs institutionnels du vin en France : études de cas. XLVII Colloque de l'ASRDLF, Aoste, 20-22 septembre.

Boyer R., 1990. Les problématiques de la régulation face aux spécificités sectorielles. Perspectives ouvertes sur la thèse de Pierre Bartoli et Daniel Boulet. Cahiers d'Économie et de Sociologie Rurales 17, 39-76.

Boyer R., 2006. Comment changent les modes de régulation sectoriels. Postface à Laferté G., La Bourgogne et ses vins : une image d'origine contrôlée, Belin.

Boyer R., Boyer D., Laferté F., 2007. La connexion des réseaux comme facteur de changement institutionnel : l'exemple des vins de Bourgogne. Working Paper 42, École d'Économie de Paris, Paris.

Brouard J., Ditter J.G., 2008. L'articulation entre terroir et territoire dans les stratégies de la filière vitivinicole française, une comparaison Chablis-Cahors. Colloque Chaire UNESCO : «De Jules Guyot à Robert Parker : 150 ans de construction des territoires du vin », Beaune, 13-15 Novembre.

Calvet J., 2005. Les clusters vitivinicoles français à AOC. Une analyse en termes de biens clubs, Revue d'Économie Régionale et Urbaine 4, 481-506.

Caron A., Torre A., 2005. Conflits d'usages et de voisinage dans les espaces ruraux. In Torre A., Filippi M. coord., Proximités et changements socioéconomiques dans les mondes ruraux, Paris, INRA, 297-314.

Commons JR., 1950. The Economics of Collective Action. University of Wisconsin Press.

Coase R., 1937. The nature of the firm. Economica 4, 386-405.

Corade N., Delhomme B. 2008. Le territoire comme fondement de l'avenir des petites A.O.C viticoles : l'exemple de l'A.O.C Pécharmant en Bergeracois. Communication au XLV Colloque de l'ASRDLF, Rimouski, 25-26-27 août.

Di Meo G., 1996. Les territoires du quotidien. L'Harmattan, Paris.

Didry C., Vincensini C., 2010. Au-delà de la dichotomie marché-institutions : l'institutionnalisme de Douglass North face au défi de Karl Polanyi. Revue Française de Socio-Économie 5, 205-224.

DiMaggio P.J., 1988. Interest and agency in institutional theory. In Zucker L.G. Ed., Institutional Patterns and Organizations Culture and Environment. Ballinger Publishing, Cambridge Mass, 3-21.

Ditter J.G., 2005. Clusters et terroirs : les systèmes productifs localisés dans la filière vinicole. Reflets et Perspectives de la Vie économique XLIV (4).

Ditter J.G., Brouard J., 2009. Stratégie, terroir et territoire vitivinicoles dans une perspective institutionnelle : le cas de l'AOC Cahors. XLVI Colloque de l'ASRDLF, Clermont-Ferrand, 6-8 juillet.

Fourastié J., 1979. Les Trente Glorieuses. Hachette, Paris.

Frayssignes J., 2005. Les AOC dans le développement territorial : Une analyse en termes d'ancrage appliquée aux cas français des filières fromagères. Thèse de doctorat, soutenue le 12 décembre, Institut National Polytechnique de Toulouse. 
Garcia-Parpet M.-F., 2001. Le terroir, le cépage et la marque : stratégies de valorisation des vins dans un contexte de mondialisation. Cahiers d'Économie et Sociologie Rurales 60-61, 25-57.

Gilly J.P., Lung Y., 2004. Proximités, secteurs, territoires. 4 e journée de la Proximité : proximités, réseaux et coordination, Marseille, juin.

Hall P., Soskice D., 2001, Varieties of Capitalism: The Institutional Foundations of Comparative Advantage, Oxford University Press.

Hinnewinkel J.-C., Le Gars C., 2002. Les territoires de la vigne et du vin. Éditions Féret, Bordeaux.

Jacquet O., 2009. Un siècle de construction du vignoble bourguignon : les organisations vitivinicoles de 1884 aux AOC. Éditions Universitaires de Dijon, Dijon.

Laferté G., 2006. La Bourgogne et ses vins : une image d'origine contrôlée. Belin, Paris.

Laporte, C., 2000. L'Appellation d'Origine Contrôlée comme garant de la typicité des productions viticoles. Revue d'Économie Régionale et Urbaine 3, 557-570.

Leloup F., Moyart L., Pecqueur B., 2005. La gouvernance territoriale comme nouveau mode de coordination territoriale. Géographie, Économie et Société 7 (4), 321-331.

Lévy J., Lussault M., 2003, Dictionnaire de la géographie et de l'espace des sociétés, Belin, Paris.

Marshall A., 1890. Principles of Economics. Macmillan and Co.

Martin J.-C., 2004. Terroir et stratégie des producteurs dans la filière vitivinicole. In D'Hauteville F. et al., Bacchus 2005, Dunod, Paris.

North D.C., 1990. Institutions, Institutional Change and Economic Performance. Cambridge, Cambridge University Press.

North D.C., 2005. Institutions and the Process Of Economic Change. Management International 9 (3), 1-7.

Olivier V., 2009. Diagnostic de la filière "AOC Cahors ». Étude réalisée par des étudiants de $3^{e}$ année de l’École Supérieure Agronomique de Toulouse ENSAT.

Pecqueur B., Zimmermann J.-B., 2004. Économie de proximités, Hermès Lavoisier, Paris.

Polge M., 2003. Petite entreprise et stratégie de terroir. Revue française de gestion 144, 181-193.

Rallet A., Torre A., 2004. Proximité et localisation. Économie Rurale 28, mars avril, 25-41.

Rastoin J.-L., Vissac-Charles V., 1999. Le groupe stratégique des entreprises de terroir. Revue internationale PME 12 (1-2), 171-192.

Rouvellac E., 2009. De la Cartographie des terroirs de l'aire AOC Cahors à la proposition d'un classement des terroirs en un «Premier Cru Cahors ». Territoires du vin, janvier http://revuesshs.u-bourgogne.fr/ territoiresduvin/document.php?id=205

Scott W.R., 1995. Institutions and Organizations. Sage, Thousand Oaks.

Steinmo S., 2010, The Evolution of Modern States: Sweden, Japan, and the United States, Cambridge University Press, Cambridge.

Thelen K., 2005, How Institutions Evolve: The Political Economy of Skills in Germany, Britain, the United States and Japan. Cambridge University Press, Cambridge.

Torre A., Filippi M., Eds. 2005. Proximité et changements socio-économiques dans les mondes ruraux, INRA Éditions, Paris

Torre A., 2009. Retour sur la notion de proximité géographique. Géographie, Economie et Société 11 (1), 63-75

Torre A., 2002. Les AOC sont-elles des Clubs? Réflexions sur les conditions de l'action collective localisée, entre coopération et règles formelles. Revue d'Économie industrielle 100, 39-62.

Tulet J.-C., Velasco-Graciet H., 2003. Refondation d'un grand vignoble du Sud de la France : le Cahors. In Casanova A. Ed., La vigne en Méditerranée occidentale, Éditions du CTHS, 103-112. http://cths.fr/ed/ edition.php?id=1223

Velasco-Graciet H., 2002. Le vin et le vignoble de Cahors à l'usage du voyageur : image, représentation et construction d'un territoire. In Hinnewinkel, J.-C., Le Gars, C., Les territoires de la vigne et du vin, Éditions Féret, bordeaux. 
Velasco-Graciet H., Rouvellac E., 2002. Le vignoble de Cahors au temps de l'économie-monde. Sud-Ouest européen 14, 87-98.

Zimmermann J.B., 2008. Le territoire dans l'analyse économique. Proximité géographique et proximité organisée, Revue française de Gestion 184, 105-118.

\section{Références complémentaires}

Amable B., 2005. Les cinq capitalismes : diversité des systèmes économiques et sociaux dans la mondialisation. Le Seuil, Paris.

César G., 2002. L'avenir de la viticulture française : entre tradition et défi du nouveau monde. Rapport d'information du Sénat 349. http://www.senat.fr/rap/r01-349/r01-349.html.

Chambolle C., Giraud Héraud E., 2002. Certification de la qualité par une AOC : un modèle d'analyse. Cahiers du LORIA 2002-01, Mai.

Marchesnay M., 2002. Individual and Collective Entrepreneurship for SMEs: the Case of "French Exception". ERFI Working Paper.

Rallet A., 2002. L'économie de proximités : propos d'étape. Etudes et Recherches sur les systèmes agraires et le développement $33,11-25$.

Torrès O., 2004. The failure of the Californian Mondavi's implantation in France: entrepreneurship and corporatism. International. Journal of Entrepreneurship and Small Business 1 (1-2), 70-99. 
